\title{
Comparison of the Efficacy of Rosuvastatin versus Atorvastatin in Preventing Contrast Induced Nephropathy in Patient with Chronic Kidney Disease Undergoing Percutaneous Coronary Intervention
}

\author{
Yong Liu' ${ }^{19}$, Yuan-hui Liu ${ }^{1,29}$, Ning Tan ${ }^{1 *}$, Ji-yan Chen ${ }^{1 *}$, Ying-ling Zhou ${ }^{1}$, Li-wen Li ${ }^{1}$, Chong-yang Duan ${ }^{3}$, \\ Ping-Yan Chen ${ }^{3}$, Jian-fang Luo ${ }^{1}$, Hua-long $\mathrm{Li}^{1}$, Wei-Guo ${ }^{1}$ \\ 1 Department of Cardiology, Guangdong Cardiovascular Institute, Guangdong General Hospital, Guangdong Academy of Medical Sciences, Guangzhou, Guangdong, \\ China, 2 Southern medical university, Guangzhou, Guangdong, China, 3 Department of Biostatistics, School of Public Health and Tropical Medicine, Southern Medical \\ University, Guangzhou, China
}

\begin{abstract}
Objectives: We prospectively compared the preventive effects of rosuvastatin and atorvastatin on contrast-induced nephropathy $(\mathrm{CIN})$ in patients with chronic kidney disease (CKD) undergoing percutaneous coronary intervention (PCI).

Methods: We enrolled 1078 consecutive patients with CKD undergoing elective PCl. Patients in Group $1(n=273)$ received rosuvastatin $(10 \mathrm{mg})$, and those in group $2(n=805)$ received atorvastatin $(20 \mathrm{mg})$. The primary end-point was the development of CIN, defined as an absolute increase in serum creatinine $\geq 0.5 \mathrm{mg} / \mathrm{dL}$, or an increase $\geq 25 \%$ from baseline within 48-72 h after contrast medium exposure.

Results: CIN was observed in $58(5.4 \%)$ patients. The incidence of CIN was similar in patients pretreated with either rosuvastatin or atorvastatin $(5.9 \%$ vs. $5.2 \%, p=0.684)$. The same results were also observed when using other definitions of CIN. Clinical and procedural characteristics did not show significant differences between the two groups ( $p>0.05$ ). Additionally, there were no significant inter-group differences with respect to in-hospital mortality rates $(0.4 \%$ vs. $1.5 \%$, $p=0.141$ ), or other in-hospital complications. Multivariate logistic regression analysis revealed that rosuvastatin and atorvastatin demonstrated similar efficacies for preventing CIN, after adjusting for potential confounding risk factors (odds ratio $=1.17,95 \%$ confidence interval, $0.62-2.20, p=0.623$ ). A Kaplan-Meier survival analysis showed that patients taking either rosuvastatin or atorvastatin had similar incidences of all-cause mortality $(9.4 \%$ vs. $7.1 \%$, respectively; $p=0.290)$ and major adverse cardiovascular events $(29.32 \%$ vs. $23.14 \%$, respectively; $p=0.135)$ during follow-up.
\end{abstract}

Conclusions: Rosuvastatin and atorvastatin have similar efficacies for preventing CIN in patients with CKD undergoing PCI.

Citation: Liu Y, Liu Y-h, Tan N, Chen J-y, Zhou Y-l, et al. (2014) Comparison of the Efficacy of Rosuvastatin versus Atorvastatin in Preventing Contrast Induced Nephropathy in Patient with Chronic Kidney Disease Undergoing Percutaneous Coronary Intervention. PLoS ONE 9(10): e111124. doi:10.1371/journal.pone. 0111124

Editor: Garyfalia Drossopoulou, National Centre for Scientific Research “Demokritos", Greece

Received June 20, 2014; Accepted September 19, 2014; Published October 30, 2014

Copyright: ( 2014 Liu et al. This is an open-access article distributed under the terms of the Creative Commons Attribution License, which permits unrestricted use, distribution, and reproduction in any medium, provided the original author and source are credited.

Data Availability: The authors confirm that all data underlying the findings are fully available without restriction. All relevant data are within the paper.

Funding: This study was supported by grant from the National Natural Science Foundation of China (grant no. 81270286). The funders had no role in the study design, data collection and analysis, decision to publish, or preparation of the manuscript; the work was not funded by any industry sponsors.

Competing Interests: The authors have declared that no competing interests exist.

*Email: tanning100@126.com (NT); cgy01973@126.com (JYC)

9 These authors contributed equally to this work.

\section{Introduction}

Contrast-induced nephropathy (CIN) is an important and wellknown complication in patients undergoing percutaneous coronary intervention (PCI). CIN also causes prolonged in-hospital stays and excess health care costs, and represents a powerful predictor of short and long term adverse outcomes [1,2,3]. CIN occurs even more frequently in patients with chronic kidney disease (CKD), with a reported incidence as high as $20-26.6 \%$ $[3,4]$. However, other than periprocedural hydration with normal saline, limiting the amount of contrast medium (CM), and using iso- or low-osmolar CM, few strategies are effective for preventing CIN.

Statins belong to a drug class that has pleiotropic effects on the vasculature and improves endothelial function, probably by increasing nitric oxide synthetase bioavailability and decreasing oxidative stress $[5,6,7]$. These properties counteract specific pathophysiologic mechanisms that promote the development of CIN $[2,8]$. In recent years, increasing evidence has supported the preventive effect of atorvastatin on CIN development in patients undergoing PCI $[9,10]$. Additionally, two large randomized control trials (RCTs) demonstrated that rosuvastatin significantly 
reduced the risk of CIN and improved short term clinical outcomes $[11,12]$. However, not all statins (especially, rosuvastatin and atorvastatin) are equivalent; they vary in several properties, including low-density lipoprotein (LDL) cholesterol lowering potency, lipophilicity, renoprotection, anti-inflammatory effects, and their effects on myocardial function $[13,14]$. Whether these differences significantly influence their effect on preventing CIN remains unknown. Recently, Kaya et al. (ROSA-CIN trial) conducted a study including 198 ST-segment elevation myocardial infarction (STEMI) patients undergoing primary PCI to determine if rosuvastatin and atorvastatin had similar efficacies for preventing CIN [15]. However, the number of enrolled patients was too small to draw definite conclusions; additional large trials are required to confirm their similarity. Therefore, we performed a prospective study to compare the preventive effects of rosuvastatin and atorvastatin on CIN in patients with CKD undergoing PCI.

\section{Patients and Methods}

\section{Patient population}

We prospectively enrolled consecutive CKD patients undergoing PCI at Guangdong Cardiovascular Institute, Guangdong General Hospital, China, between March 2010 and September 2012. The inclusion criteria included: patients with an estimated glomerular filtration rate (eGFR) of $30-90 \mathrm{~mL} / \mathrm{min} / 1.73 \mathrm{~m}^{2}$ (CKD stages II and III), and patients pretreated with either atorvastatin $(20 \mathrm{mg})$ or rosuvastatin $(10 \mathrm{mg})$, at equivalent standard doses [16]. Statin pretreatment was defined as taking a statin 2-3 days before CM exposure and 2-3 days after the procedure. Patients were excluded if they had undergone chronic statin therapy ( $>14$ days); had been treated with simvastatin or other statins; had a history of heart failure (defined as NYHA III/ IV or Killip class II-IV), pregnancy, CM allergy, CM exposure during the previous 7 days; or had been treated with potentially nephroprotective (e.g., N-acetylcysteine or theophylline) or nephrotoxic (e.g., steroids, non-steroidal anti-inflammatory drugs, aminoglycosides, amphotericin B) drugs [17]. We also excluded patients with CKD stages 0 , IV or V; hepatic insufficiency; or who had undergone renal transplantation or dialysis.

This study protocol was approved by the Guangdong General Hospital ethics committee and the study conformed to the Declaration of Helsinki. Written informed consent was obtained from all patients before the procedure.

\section{Biochemical investigations}

Serum creatinine ( $\mathrm{SCr}$ ) levels were measured upon admission and within 48-72 h after CM exposure. Blood urea nitrogen (BUN), creatine kinase MB, fasting glucose, electrolytes, fasting lipid profiles, albumin, and other standard clinical parameters were measured in the morning before the procedure. The eGFR was evaluated using the 4-variable Modification of Diet in Renal Disease equation based on Chinese patients [18]. Left ventricular function was echocardiographically evaluated in each patient within a 24-h period before the PCI.

\section{$\mathrm{PCl}$ and medications}

PCI was performed by experienced interventional cardiologists according to standard clinical practice using standard techniques. Nonionic, low-osmolar CM was used in all patients (either Iopamiron or Ultravist, both at $370 \mathrm{mg} \mathrm{I} / \mathrm{mL}$ ). Normal saline $(0.9 \%)$ at a rate of $1 \mathrm{~mL} / \mathrm{kg} / \mathrm{h}(0.5 \mathrm{~mL} / \mathrm{kg} / \mathrm{h}$ if the patient's left ventricular ejection fraction (LVEF) was $<40 \%$ ) was administered intravenously $3-12 \mathrm{~h}$ before and $6-12 \mathrm{~h}$ after CM exposure. Antiplatelet agents (aspirin/clopidogrel), $\beta$-adrenergic blocking agents, statins, diuretics, angiotensin-converting enzyme inhibitors, and inotropic drugs were used at the attending cardiologist's discretion, according to clinical protocols derived from interventional guidelines.

\section{Clinical outcomes}

Follow-up events were carefully monitored and recorded by trained nurses through office visits and telephone interviews conducted, at 1, 6, 12, and 24 months after cardiac catherization.

The primary end-point was CIN development, defined as an absolute increase in $\mathrm{SCr} \geq 0.5 \mathrm{mg} / \mathrm{dL}$ or a relative increase $\geq 25 \%$ from baseline, within 48-72 h after CM exposure. Additional end points included: CIN, as defined by other criteria [17], and major in-hospital or long-term adverse clinical events (MACEs), including all-cause mortality, non-fatal myocardial infarction, target vessel revascularization, CIN requiring renal replacement therapy, and stroke.

The other CIN definitions included: an absolute increase in SCr of $\geq 0.5 \mathrm{mg} / \mathrm{dL}$ within $48-72 \mathrm{~h}$ (CIN2); an absolute increase in $\mathrm{SCr}$ of $\geq 0.3 \mathrm{mg} / \mathrm{dL}$ within $48 \mathrm{~h}$ (CIN3); a SCr increase of $\geq 50 \%$ (1.5 fold from baseline) within $48 \mathrm{~h}$ (CIN4); and CIN5 (CIN3 or CIN4) [17].

\section{Statistical analysis}

SAS version 9.2 (SAS Institute, Cary, NC, USA) was used for all analyses. Continuous variables are described as means $\pm \mathrm{SD}$ or medians, and categorical variables as absolute values (percentages). Comparisons of between-groups differences were performed using Student's $t$-test or the Wilcoxon rank sum test (if not normally distributed) for continuous variables and a chi-square or Fisher's exact test for categorical variables. Logistic regression analysis was performed using CIN as the dependent variable. Variables that were statistically significant according to a univariate analysis, were included in the final multivariate model to identify CIN predictors. Cumulative event curves for both groups of patients were created using the Kaplan-Meier survival method and compared using the log-rank test. All statistical tests were 2-tailed and statistical significance was inferred if $\mathrm{P}<0.05$.

\section{Results}

\section{Baseline characteristics between patients pretreated with atorvastatin and rosuvastatin}

A total of 1078 consecutive CKD patients, pretreated with atorvastatin or rosuvastatin were analyzed (mean age, 65.2 \pm 10.1 years; mean eGFR, $69.8 \pm 14.0 \mathrm{~mL} / \mathrm{min} / 1.73 \mathrm{~m}^{2}$; mean Mehran

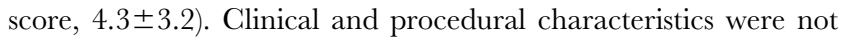
significantly different between the two groups. In particular, the proportions of patients with diabetes mellitus (DM, $\mathrm{P}=0.091)$, age $\geq 75$ years $(P=0.200)$, or anemia $(P=0.187)$ were similar in both groups. The baseline $\mathrm{SCr}(\mathrm{P}=0.495)$ and eGFR $(\mathrm{P}=0.704)$ levels were also similar between the two groups, as were the mean LVEF (rosuvastatin $59.96 \pm 11.18 \%$ vs. atorvastatin $59.05 \pm 11.77 \%$, $\mathrm{P}=0.291$ ), $\mathrm{CM}$ volumes used (rosuvastatin 133.36 $\pm 67.75 \mathrm{~mL}$ vs. atorvastatin $132.37 \pm 70.13 \mathrm{~mL}, \mathrm{P}=0.838)$, and Mehran risk scores (rosuvastatin $4.06 \pm 2.86$ vs. atorvastatin 4.42 \pm 3.31 , $\mathrm{P}=0.095$ ). (Table 1).

\section{Preventive effect of statins on CIN and in hospital outcomes}

Overall, CIN was observed in 58 patients (5.4\%). Compared with patients without CIN, patients with CIN had a significantly higher rate of in-hospital mortality $(10.34 \%$ vs. $0.69 \%, \mathrm{P}<0.001)$, 
Table 1. Baseline clinical characteristics of study participants.

\begin{tabular}{|c|c|c|c|}
\hline Variables & Rosuvastatin ( $n=273$ ) & Atorvastatin $(\mathbf{n}=\mathbf{8 0 5})$ & $\mathbf{P}$ \\
\hline \multicolumn{4}{|l|}{ Demographics } \\
\hline Age, (y) & $65.28 \pm 9.89$ & $65.79 \pm 10.28$ & 0.425 \\
\hline Age $>75$ y, (\%) & $36(13.2 \%)$ & $126(15.7 \%)$ & 0.443 \\
\hline Females (\%) & $57(20.9 \%)$ & $187(23.2 \%)$ & 0.423 \\
\hline Weight (kg) & $65.58 \pm 10.18$ & $65.17 \pm 10.24$ & 0.409 \\
\hline $\mathrm{SBP}(\mathrm{mmHg})$ & $133.07 \pm 21.64$ & $131.01 \pm 20.44$ & 0.158 \\
\hline $\mathrm{DBP}(\mathrm{mmHg})$ & $76.64 \pm 11.44$ & $75.31 \pm 11.23$ & 0.093 \\
\hline Heart rate $(\mathrm{bpm})$ & $74.33 \pm 12.32$ & $72.94 \pm 12.15$ & 0.105 \\
\hline \multicolumn{4}{|l|}{ Medical history, n (\%) } \\
\hline Smokers & 108(39.6\%) & $301(37.4 \%)$ & 0.523 \\
\hline Hypertension & $176(64.5 \%)$ & $506(62.9 \%)$ & 0.633 \\
\hline Diabetes & $56(20.5 \%)$ & $206(25.6 \%)$ & 0.091 \\
\hline Dyslipidemia & $41(15.0 \%)$ & $112(13.9 \%)$ & 0.651 \\
\hline Prior MI & $31(11.4 \%)$ & $100(12.4 \%)$ & 0.641 \\
\hline Prior CABG & $4(1.5 \%)$ & $8(1.0 \%)$ & 0.521 \\
\hline \multicolumn{4}{|l|}{ Laboratory findings } \\
\hline Baseline $\mathrm{SCr}(\mu \mathrm{mol} / \mathrm{L})$ & $99.29 \pm 24.77$ & $98.17 \pm 23.07$ & 0.495 \\
\hline Baseline-eGFR DDEeGFR $\left(\mathrm{mL} / \mathrm{min} / 1.73 \mathrm{~m}^{2}\right)$ & $69.49 \pm 14.83$ & $69.86 \pm 13.73$ & 0.704 \\
\hline Log-NT-pro-BNP (pg/mL) & $5.59 \pm 1.76$ & $5.66 \pm 1.68$ & 0.573 \\
\hline hs-CRP (mg/L) & $12.02 \pm 21.96$ & $10.10 \pm 19.77$ & 0.281 \\
\hline LVEF, \% & $59.96 \pm 11.18$ & $59.05 \pm 11.77$ & 0.291 \\
\hline Total cholesterol (mmol/L) & $4.23 \pm 1.08$ & $4.29 \pm 1.94$ & 0.660 \\
\hline Triglyceride $(\mathrm{mmol} / \mathrm{L})$ & $1.44 \pm 0.89$ & $1.79 \pm 8.17$ & 0.329 \\
\hline LDL (mmol/L) & $2.53 \pm 0.94$ & $2.48 \pm 0.86$ & 0.548 \\
\hline HbA1c, \% & $6.53 \pm 1.53$ & $6.49 \pm 1.20$ & 0.679 \\
\hline$H G, g / L$ & $132.21 \pm 14.77$ & $132.57 \pm 16.54$ & 0.733 \\
\hline Anemia, $n(\%)$ & $86(31.5 \%)$ & 289(35.9\%) & 0.187 \\
\hline Serum albumin, $g / L$ & $34.76 \pm 3.95$ & $35.47 \pm 4.29$ & 0.018 \\
\hline Uric acid, $\mu \mathrm{mol} / \mathrm{L}$ & $374.81 \pm 103.30$ & $389.95 \pm 108.719$ & 0.074 \\
\hline \multicolumn{4}{|l|}{ Medication, n (\%) } \\
\hline ACEI/ARB & $242(88.6 \%)$ & 729(90.6\%) & 0.361 \\
\hline$\beta$-bloker & $237(86.8 \%)$ & $720(89.4 \%)$ & 0.235 \\
\hline Calcium channel blocker & $70(25.6 \%)$ & $163(20.2 \%)$ & 0.061 \\
\hline Diuretics & $27(9.9 \%)$ & $101(12.5 \%)$ & 0.241 \\
\hline \multicolumn{4}{|l|}{ Procedural characteristic } \\
\hline Contrast volume $(\mathrm{mL})$ & $133.36 \pm 67.75$ & $132.37 \pm 70.13$ & 0.838 \\
\hline Contrast exposure time $(\mathrm{min})(\mathrm{min})$ & $73.37 \pm 43.97$ & $71.96 \pm 47.34$ & 0.669 \\
\hline Number of diseased vessels ( $n$ ) & $2.14 \pm 1.05$ & $2.03 \pm 1.12$ & 0.156 \\
\hline Number of stents $(n)$ & $1.68 \pm 1.20$ & $1.60 \pm 1.19$ & 0.387 \\
\hline Contrast volume/eGFR ratio & $2.07 \pm 1.28$ & $2.01 \pm 1.22$ & 0.467 \\
\hline Mehran score & $4.06 \pm 2.86$ & $4.42 \pm 3.31$ & 0.095 \\
\hline
\end{tabular}

Abbreviations: SBP: systolic blood pressure; DBP: diastolic blood pressure. MI: myocardial infarction; CABG: coronary artery bypass grafting; SCr: serum creatinine; eGFR: estimated glomerular filtration rate; NT-pro-BNP: N-Terminal Pro-B-Type natriuretic peptide; hs-CRP: high sensitivity C reactive protein; LVEF: left ventricular ejection fraction; LDL: low density lipoprotein; HbA1c: hemoglobin A1c; HG: hemoglobin: ACEl/ARB: angiotensin-converting enzyme inhibitor/angiotensin receptor blocker;

Mehran score: model to define contrast-induced nephropathy (CIN) by Mehran et al. Anemia was defined using World Health Organization criteria: baseline hematocrit value $<39 \%$ for men and $<36 \%$ for women.

doi:10.1371/journal.pone.0111124.t001

and other in hospital complications, such as the requirement for renal replacement therapy $(3.4 \%$ vs. $0.4 \%, \mathrm{P}=0.002)$ and the use of intra-aortic balloon pump (IABP; $10.34 \%$ vs. $1.18 \%, \mathrm{P}<0.001$ ). (Figure 1).
The incidences of CIN were similar between patients pretreated with either rosuvastatin or atorvastatin $(5.9 \%$ vs. $5.2 \%, \mathrm{P}=0.684)$; similar results were also obtained using the alternate CIN definitions. In addition, there were no significant differences 


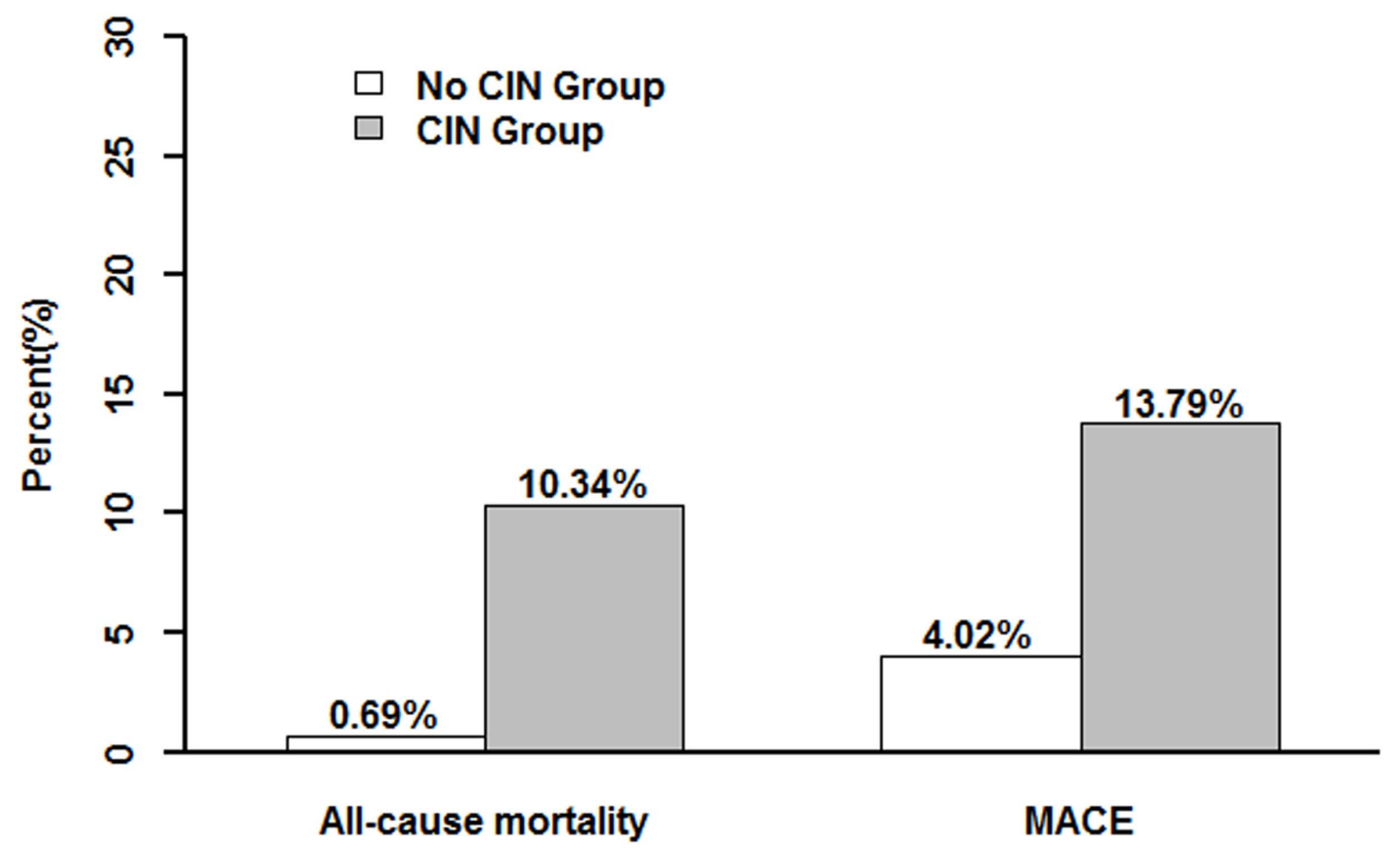

Figure 1. Multivariate logistic analysis associating contrast-induced nephropathy with various risk indicators. doi:10.1371/journal.pone.0111124.g001

between the two groups with regard to the rate of in-hospital mortality $(0.4 \%$ vs. $1.5 \%, \mathrm{P}=0.141)$. However, patients treated with rosuvastatin had a lower incidence of in-hospital MACEs than those treated with atorvastatin $(1.8 \%$ vs. $5.5 \%, \mathrm{P}=0.013)$ (Table2).

Multivariate logistic regression analysis revealed that pretreatment with rosuvastatin had a similar effect as atorvastatin pretreatment regarding the development of CIN in patients undergoing PCI (odds ratio $[\mathrm{OR}]=1.17,95 \%$ confidence interval [CI], 0.62-2.20, $\mathrm{P}=0.623$ ), even after adjusting for potential confounding risk factors (age $>75$ years, eGFR $\leq 60 \mathrm{~mL} / \mathrm{min}$ / $1.73 \mathrm{~m}^{2}$, DM, anemia, $\mathrm{CM}>100 \mathrm{~mL}$, IABP, $\mathrm{LVEF}<40 \%$, primary PCI). Age $>75$ years $(\mathrm{P}=0.029)$, IABP $(\mathrm{P}=0.023)$, and primary PCI $(\mathrm{P}=0.007)$ were other independent predictors of CIN in this population. (Figure 2).

\section{Clinical outcomes during follow-up}

The median follow-up period was $2.51 \pm 0.86$ years (inter quartile range, $1.80-3.27$ years) and was continued for all patients who survived to discharge.

To determine the relationship between the accumulated risk of adverse events and rosuvastatin or atorvastatin pretreatment, a Kaplan-Meier survival analysis was performed. Patients pretreated either rosuvastatin or atorvastatin demonstrated a similar incidence of all-cause mortality $(7.76 \%$ vs. $5.36 \%, \mathrm{P}=0.193)$ or MACEs $(26.48 \%$ vs. $21.28 \%, \mathrm{P}=0.243)$, as illustrated in Figure 3. In addition, patients who developed CIN had a higher rate of allcause mortality than those who did not (cumulative rate of mortality, $22.73 \%$ vs. $5.07 \%, \mathrm{P}<0.001)$. A similar result was found for MACEs. (43.18\% vs. 21.50\%, P=0.002). (Figure 4).

Table 2. In-hospital events in patients treated with rosuvastatin or atorvastatin.

\begin{tabular}{|c|c|c|c|}
\hline Variables & Rosuvastatin $(n=273$ ) & Atorvastatin $(n=805)$ & $\mathbf{P}$ \\
\hline CIN1 & $16(5.9 \%)$ & $42(5.2 \%)$ & 0.684 \\
\hline CIN2 & $5(1.8 \%)$ & $13(1.6 \%)$ & 0.809 \\
\hline CIN3 & $10(3.7 \%)$ & $33(4.1 \%)$ & 0.750 \\
\hline CIN4 & $2(0.7 \%)$ & $10(1.2 \%)$ & 0.488 \\
\hline CIN5 & $10(3.7 \%)$ & $33(4.1 \%)$ & 0.750 \\
\hline Death & $1(0.4 \%)$ & $12(1.5 \%)$ & 0.141 \\
\hline Renal replacement therapy & $1(0.4 \%)$ & $5(0.6 \%)$ & 0.625 \\
\hline Hypotension & $3(1.1 \%)$ & $16(2.0 \%)$ & 0.335 \\
\hline IABP & $3(1.1 \%)$ & 15 (1.9\%) & 0.394 \\
\hline Acute heart failure & $2(0.7 \%)$ & $11(1.4 \%)$ & 0.407 \\
\hline Cerebrovascular accident & $0(0.0 \%)$ & $3(0.4 \%)$ & 0.312 \\
\hline
\end{tabular}

Abbreviations: CIN: contrast induced nephropathy; IABP: intra-aortic ballon pump.

doi:10.1371/journal.pone.0111124.t002 


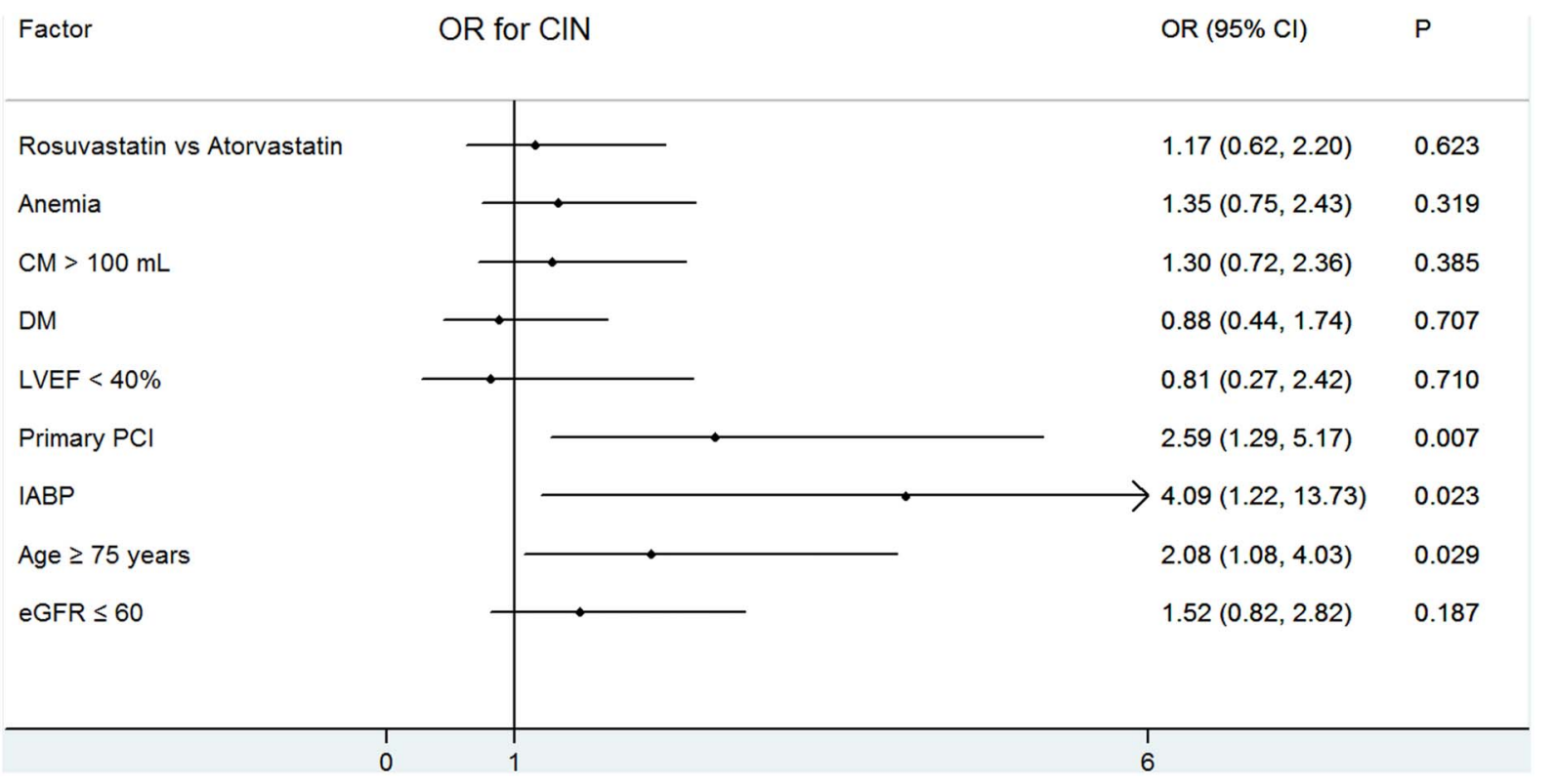

Figure 2. The prevalence of in-hospital all-cause mortality or major adverse cardiovascular events in patients with or without contrast-induced nephropathy. doi:10.1371/journal.pone.0111124.g002

\section{Discussion}

The present study may be the first to demonstrate that pretreatments with either rosuvastatin or atorvastatin have similar efficacies for preventing CIN in patients with CKD undergoing PCI.

The prevention of CIN is an important concern because it affects patient morbidity and mortality, especially in CKD patients $[3,4]$. In the current study, we found that the incidence of CIN was $5.4 \%$, in agreement with previous studies [3]. Similar to previous studies, we found that patients developing CIN had a higher risk of poor in-hospital and long-term clinical outcomes. Because, few strategies have been demonstrated to be effective for preventing CIN [17]. The development of new strategies to decrease CIN occurrence, especially for high-risk CKD patients is urgently needed. This has led to an increased interest in the preventive

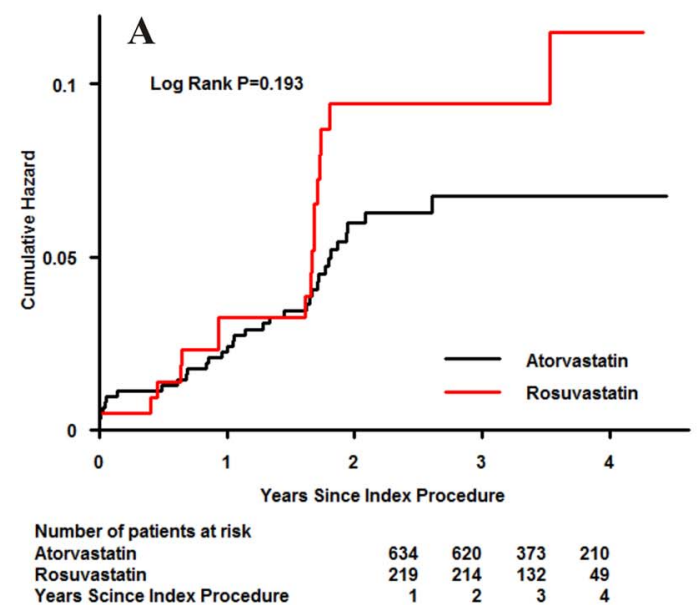

effects of statins (especially, atorvastatin and rosuvastatin) on CIN development in patients undergoing PCI.

However, conflicting results have been published. Kandula et al [19] reported an observational study (239 patients with statins, 114 without statins), that showed statin treatment was not associated with CIN prevention, after adjusting for the propensity of receiving statins $(\mathrm{OR}=1.6,95 \%$ CI: $0.86-3.22, \mathrm{P}=0.12)$. In contrast, another study based on a database of 29,409 patients undergoing emergent and non-emergent PCI [20], reported that patients using statins had a lower risk of CIN than did those not using statins $(4.4 \%$ vs. $5.9 \%, \mathrm{P}<0.001)$. Similar results were demonstrated by Hoshi et al [21]. Other than these observational studies, many RCTs have been conducted to address this topic. Toso et al [22] performed a prospective RCT, including 304 patients, to investigate the efficacy of short-term high dose atorvastatin on preventing CIN development in patients with

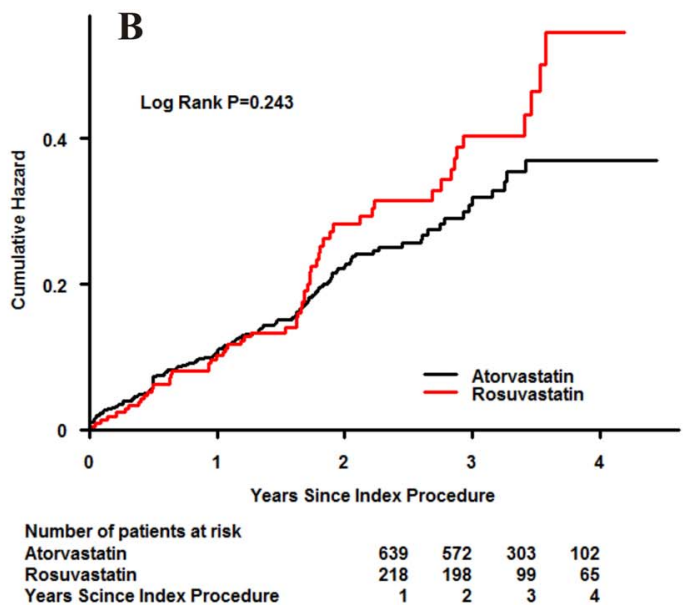

Figure 3. Cumulative rate of follow-up all-cause mortality (A) or major adverse cardiovascular events (B) in patients initially treated with rosuvastatin or atorvastatin. doi:10.1371/journal.pone.0111124.g003 

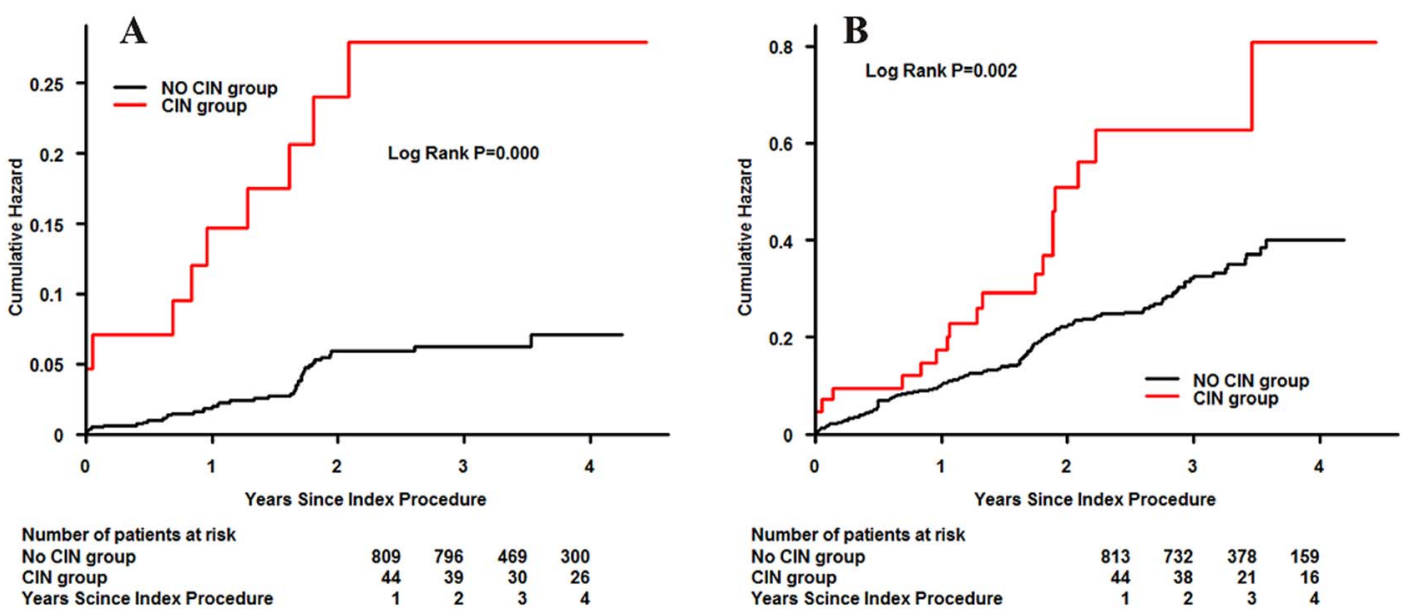

Figure 4. Cumulative rate of follow-up all-cause mortality (A) or major adverse cardiovascular events (B) in patients with or without contrast-induced nephropathy.

doi:10.1371/journal.pone.0111124.g004

CKD undergoing PCI. The results showed that short-term high doses of atorvastatin, administered periprocedurally, did not decrease CIN occurrence in patients with pre-existing CKD. However, another group [10] enrolled 410 patients with GKD in an RCT and demonstrated that a single high dose of atorvastatin administered within a $24 \mathrm{~h}$ period before CM exposure, was effective at reducing the CIN rate. Similar findings have been reported from subsequent RCTs [9,21,23]. A previous metaanalysis of 7 RCTs, with a total of 1399 patients (693 patients receiving high-dose statins, 706 receiving low-dose or no statins) revealed that atorvastatin was beneficial for preventing of CIN [24], which is in agreement with our recent meta-analysis [25].

Two large RCTs recently demonstrated that rosuvastatin pretreatment, upon admission, could reduce CIN occurrence in patients undergoing PCI. Leoncini et al [11] reported that acute coronary syndrome patients, without ST-segment elevation, who were treated with rosuvastatin $(40 \mathrm{mg}$ on-admission, followed by $20 \mathrm{mg}$ /day) experienced less CIN than patients not receiving rosuvastatin. Similarly, in patients with type $2 \mathrm{DM}$ and GKD, another group showed that rosuvastatin significantly reduced the risk of CIN after CM exposure [12]. Accordingly, although guideline committees have not recommended this CIN-prevention strategy, researchers are increasingly considering statins as an effective drug for preventing CIN, based on the existing evidence.

Although the mechanism of statins in CIN prevention remains unknown, the following mechanisms may play important roles. In addition to their intended impact on blood cholesterol levels, statins are also known to have pleiotropic effects. Previous studies showed that statins treatment could prevent renal tubular cell apoptosis and increase survival signaling pathways [10]. However, the direct toxic effects of CM on renal cells, leading cell necrosis or apoptosis, are thought to contribute to the CIN pathogenesis. Preventing CM-induced renal cell apoptosis seems to play an important role in the statins' effects on CIN [10]. In addition, endothelial dysfunction, another major contributor to CIN progression, is caused by a nitric oxide (NO) and endothelin-1 imbalance, after CM exposure. Statins may help correct this imbalance by increasing NO production and reducing endothelin1 synthesis [26]. Furthermore, G-reactive protein (CRP), as a marker of systemic inflammation, is also associated with CIN, and patients with high periprocedural GRP levels are at high risk for developing CIN $[9,27,28]$. Recent studies have demonstrated that the preventive effect of statins on CIN development parallels a significant decrease in post-procedural CRP levels [12]. Thus, statins may reduce inflammation by inhibiting pro-inflammatory mediator synthesis [29], and may have a reno-protective effect during CM exposure by attenuating inflammatory responses.

Different statins (e.g., atorvastatin and rosuvastatin) vary in their LDL-lowering potency, lipophilicity, reno-protection, and antiinflammatory effects $[13,14]$. However, whether the difference (hydrophilic and lipophilic) between statins influences their ability to reduce CIN risk is unclear. Rosuvastatin, a hydrophilic statin, has acute pleiotropic effects, and has been demonstrated to reduce LDL more aggressively, without increasing complications, and improve patient prognosis better than the other statins [30]; it also, exerts a beneficial reno-protective effect in patients with renal dysfunction [31]. Additionally, rosuvastatin has a longer plasma half-life and stronger anti-inflammatory effects than atorvastatin $[32,33]$. Because patients with CKD have significantly higher mean CRP levels [34], rosuvastatin may be more effective in these patients. Furthermore, Thiago et al demonstrated that rosuvastatin performed better than atorvastatin or simvastatin, in an experimental murine model of cigarette smoke-induced acute lung inflammation, because of better attenuation of both inflammation and oxidative stress parameters [35]. A recent meta-analysis reported that rosuvastatin might also increase apolipoprotein A-I levels at all doses more than atorvastatin [36]; apolipoprotein A-I can stabilize lipoprotein structure and has anti-inflammatory and antioxidant properties [37]. Based on these difference between rosuvastatin and atorvastatin, we hypothesized that rosuvastatin would differ from atorvastatin with respect to their abilities to prevent CIN.

To date, large studies investigating the CIN-risk reduction differences between rosuvastatin and atorvastatin have not been reported. One recent study, including 192 patients (94 taking rosuvastatin, 98 taking atorvastatin), compared the effects of different statins on CIN in STEMI patients treated with primary PCI; both statins had similar efficacies for preventing CIN. The study also suggested that the incidence of Killip class $\geq 2$ patients ranged from 91.8-94.7\% [15]. Therefore, increased of SCr in those patients may be the result of hemodynamic compromise due to acute impairment of cardiac pump function after extended myocardial infarction, rather than the direct effect of CM exposure [38]. However, in our study, the patients had relatively stable hemodynamic status because patients with a history of heart failure (NYHA $\geq$ III and Killip $\geq$ II) were excluded. Thus, CM 
administration may play a major role and the reduced risk of CIN may be a true reflection of the statins' effects. In our study, patients receiving rosuvastatin displayed higher levels of hs-CRP than did those treated with atorvastatin, suggesting that these patients would be more likely to develop CIN, based on the previous evidence $[27,28]$. However, our findings demonstrated that the incidence of CIN in rosuvastatin-treated patients was similar to that in atorvastatin- treated patients; the patients were relatively well balanced with respect to their baseline clinical and angiography characteristics. Although we did not demonstrate that rosuvastatin was superior to atorvastatin for preventing CIN, the results may not be surprising considering that different factors are involved in CIN development and that different pathophysiological mechanisms coexist.

The present study also demonstrated the patients pretreated with rosuvastatin or atorvastatin had similar risks of all-cause mortality and MACEs. In addition, we demonstrated that age $>$ 75 years, IABP use, and primary PCI were independent risk factors of CIN, but not an eGFR $\leq 60 \mathrm{~mL} / \mathrm{min} / 1.73 \mathrm{~m}^{2}$. However, Ando et al have demonstrated that eGFR as a continuous variable was a risk factor for CIN in STEMI patients treated with primary PCI [39]. This might be related to the different patient populations included in the two studies.

\section{Limitations}

There are several limitations to this study. First, this was a prospective, observational study conducted at a single center. Therefore, causality cannot be ascribed. Second, our study

\section{References}

1. Wi J, Ko YG, Kim JS, Kim BK, Choi D, et al. (2011) Impact of contrastinduced acute kidney injury with transient or persistent renal dysfunction on long-term outcomes of patients with acute myocardial infarction undergoing percutaneous coronary intervention. Heart 97: 1753-1757.

2. Seeliger E, Sendeski M, Rihal CS, Persson PB (2012) Contrast-induced kidney injury: mechanisms, risk factors, and prevention. Eur Heart J 33: 2007-2015.

3. Tsai TT, Patel UD, Chang TI, Kennedy KF, Masoudi FA, et al. (2014) Contemporary Incidence, Predictors, and Outcomes of Acute Kidney Injury in Patients Undergoing Percutaneous Coronary Interventions: Insights From the NCDR Cath-PCI Registry. JACC Cardiovasc Interv 7: 1-9.

4. Dangas G, Iakovou I, Nikolsky E, Aymong ED, Mintz GS, et al. (2005) Contrast-induced nephropathy after percutaneous coronary interventions in relation to chronic kidney disease and hemodynamic variables. Am J Cardiol 95: 13-19.

5. Wassmann S, Faul A, Hennen B, Scheller B, Bohm M, et al. (2003) Rapid effect of 3-hydroxy-3-methylglutaryl coenzyme a reductase inhibition on coronary endothelial function. Circ Res 93: e98-e103.

6. Ongini E, Impagnatiello F, Bonazzi A, Guzzetta M, Govoni M, et al. (2004) Nitric oxide (NO)-releasing statin derivatives, a class of drugs showing enhanced antiproliferative and antiinflammatory properties. Proc Natl Acad Sci U S A 101: $8497-8502$.

7. Luvai A, Mbagaya W, Hall AS, Barth JH (2012) Rosuvastatin: a review of the pharmacology and clinical effectiveness in cardiovascular disease. Clin Med Insights Cardiol 6: 17-33.

8. Wong PC, Li Z, Guo J, Zhang A (2012) Pathophysiology of contrast-induced nephropathy. Int J Cardiol 158: 186-192.

9. Patti G, Ricottini E, Nusca A, Colonna G, Pasceri V, et al. (2011) Short-term, high-dose Atorvastatin pretreatment to prevent contrast-induced nephropathy in patients with acute coronary syndromes undergoing percutaneous coronary intervention (from the ARMYDA-CIN [atorvastatin for reduction of myocardial damage during angioplasty-contrast-induced nephropathy] trial. Am J Cardiol 108: $1-7$.

10. Quintavalle G, Fiore D, De Micco F, Visconti G, Focaccio A, et al. (2012) Impact of a high loading dose of atorvastatin on contrast-induced acute kidney injury. Circulation 126: 3008-3016.

11. Leoncini M, Toso A, Maioli M, Tropeano F, Villani S, et al. (2014) Early highdose rosuvastatin for contrast-induced nephropathy prevention in acute coronary syndrome: Results from the PRATO-ACS Study (Protective Effect of Rosuvastatin and Antiplatelet Therapy On contrast-induced acute kidney injury and myocardial damage in patients with Acute Coronary Syndrome). J Am Coll Cardiol 63: 71-79.

12. Han Y, Zhu G, Han L, Hou F, Huang W, et al. (2014) Short-term rosuvastatin therapy for prevention of contrast-induced acute kidney injury in patients with diabetes and chronic kidney disease. J Am Coll Cardiol 63: 62-70. population was limited to CKD (stage II and III) patients, so the results may not extend to patients with other stage of CKD or those without CKD. Third, due to variations in the timing of measurements, we may have missed the post-procedural SCr peak. Furthermore, we did not use cystatin $\mathrm{C}$ which is a more sensitive biomarker and increases faster than SCr after CIN. Thus, the true incidence of CIN may have been underestimated. Fourth, SCr levels were not systematically measured during the follow-up period. Fifth, in consideration of previous studies revealed that high-dose atorvastatin (40 or $80 \mathrm{mg}$ ) pretreatment was more effective than low-dose $(20 \mathrm{mg})$ or no statin therapy [24], we did not investigate the protective efficacies of different doses in our study.

\section{Conclusions}

Our study demonstrated that rosuvastatin pretreatment exerts an effect similar to atorvastatin in preventing CIN in high risk patients with CKD undergoing PCI. Thus, future head to head studies are required to compare hydrophilic and lipophilic statins to determine if they reduce CIN risks differently.

\section{Author Contributions}

Conceived and designed the experiments: YL YHL NT JYG. Performed the experiments: YL YHL YLZ LWL JFL HLL WG. Analyzed the data: YL YHL CYD PYC. Contributed to the writing of the manuscript: YHL YL. Contributed to revising manuscript critically for important intellectual content: NT JYG.

13. Toth PP (2014) An update on the benefits and risks of rosuvastatin therapy. Postgrad Med 126: 7-17

14. DiNicolantonio JJ, Lavie CJ, Serebruany VL, O'Keefe JH (2013) Statin wars: the heavyweight match-atorvastatin versus rosuvastatin for the treatment of atherosclerosis, heart failure, and chronic kidney disease. Postgrad Med 125: 716.

15. Kaya A, Kurt M, Tanboga IH, Isik T, Ekinci M, et al. (2013) Rosuvastatin versus atorvastatin to prevent contrast induced nephropathy in patients undergoing primary percutaneous coronary intervention (ROSA-cIN trial). Acta Cardiol 68: 489-494.

16. Jones PH, Davidson MH, Stein EA, Bays HE, McKenney JM, et al. (2003) Comparison of the efficacy and safety of rosuvastatin versus atorvastatin, simvastatin, and pravastatin across doses (STELLAR* Trial). Am J Cardiol 92: $152-160$.

17. Stacul F, van der Molen AJ, Reimer P, Webb JA, Thomsen HS, et al. (2011) Contrast induced nephropathy: updated ESUR Contrast Media Safety Committee guidelines. Eur Radiol 21: 2527-2541.

18. Ma YC, Zuo L, Chen JH, Luo O, Yu XO, et al. (2006) Modified glomerular filtration rate estimating equation for Chinese patients with chronic kidney disease. J Am Soc Nephrol 17: 2937-2944.

19. Kandula P, Shah R, Singh N, Markwell SJ, Bhensdadia N, et al. (2010) Statins for prevention of contrast-induced nephropathy in patients undergoing nonemergent percutaneous coronary intervention. Nephrology (Carlton) 15: 165170 .

20. Khanal S, Attallah N, Smith DE, Kline-Rogers E, Share D, et al. (2005) Statin therapy reduces contrast-induced nephropathy: an analysis of contemporary percutaneous interventions. Am J Med 118: 843-849.

21. Hoshi T, Sato A, Kakefuda Y, Harunari T, Watabe H, et al. (2014) Preventive effect of statin pretreatment on contrast-induced acute kidney injury in patients undergoing coronary angioplasty: Propensity score analysis from a multicenter registry. Int J Cardiol 171: 243-249.

22. Toso A, Maioli M, Leoncini M, Gallopin M, Tedeschi D, et al. (2010) Usefulness of atorvastatin $(80 \mathrm{mg})$ in prevention of contrast-induced nephropathy in patients with chronic renal disease. Am J Cardiol 105: 288-292.

23. Li W, Fu X, Wang Y, Li X, Yang Z, et al. (2012) Beneficial effects of high-dose atorvastatin pretreatment on renal function in patients with acute ST-segment elevation myocardial infarction undergoing emergency percutaneous coronary intervention. Cardiology 122: 195-202.

24. Li Y, Liu Y, Fu L, Mei C, Dai B (2012) Efficacy of short-term high-dose statin in preventing contrast-induced nephropathy: a meta-analysis of seven randomized controlled trials. PLoS One 7: e34450.

25. Liu YH, Liu Y, Duan CY, Tan N, Chen JY, et al. (2014) Statins for the Prevention of Contrast-Induced Nephropathy After Coronary Angiography/ Percutaneous Interventions: A Meta-analysis of Randomized Controlled Trials. 
Journal ofCardiovascular Pharmacology and Therapeutics. pii: 1074248414549462. [Epub ahead of print].

26. Almuti K, Rimawi R, Spevack D, Ostfeld RJ (2006) Effects of statins beyond lipid lowering: potential for clinical benefits. Int J Cardiol 109: 7-15.

27. Liu YH, Liu Y, Tan N, Chen JY, Chen J, et al. (2014) Predictive value of GRACE risk scores for contrast-induced acute kidney injury in patients with STsegment elevation myocardial infarction before undergoing primary percutaneous coronary intervention. Int Urol Nephrol 46: 417-426.

28. Liu Y, Tan N, Zhou YL, Chen YY, Chen JY, et al. (2012) High-sensitivity Creactive protein predicts contrast-induced nephropathy after primary percutaneous coronary intervention. J Nephrol 25: 332-340.

29. Tawfik MK, Ghattas MH, Abo-Elmatty DM, Abdel-Aziz NA (2010) Atorvastatin restores the balance between pro-inflammatory and anti-inflammatory mediators in rats with acute myocardial infarction. Eur Rev Med Pharmacol Sci 14: 499-506.

30. Betteridge DJ, Gibson JM, Sager PT (2007) Comparison of effectiveness of rosuvastatin versus atorvastatin on the achievement of combined C-reactive protein $(<2 \mathrm{mg} / \mathrm{L})$ and low-density lipoprotein cholesterol $(<70 \mathrm{mg} / \mathrm{dl})$ targets in patients with type 2 diabetes mellitus (from the ANDROMEDA study). Am J Cardiol 100: 1245-1248.

31. Ridker PM, MacFadyen J, Cressman M, Glynn RJ (2010) Efficacy of rosuvastatin among men and women with moderate chronic kidney disease and elevated high-sensitivity C-reactive protein: a secondary analysis from the JUPITER (Justification for the Use of Statins in Prevention-an Intervention Trial Evaluating Rosuvastatin) trial. J Am Coll Cardiol 55: 1266-1273.

32. Qu HY, Xiao YW, Jiang GH, Wang ZY, Zhang Y, et al. (2009) Effect of atorvastatin versus rosuvastatin on levels of serum lipids, inflammatory markers and adiponectin in patients with hypercholesterolemia. Pharm Res 26: 958-964.
33. Herregods MC, Daubresse JC, Michel G, Lamotte M, Vissers E, et al. (2008) Discovery Belux: comparison of rosuvastatin with atorvastatin in hypercholesterolaemia. Acta Cardiol 63: 493-499.

34. Fox ER, Benjamin EJ, Sarpong DF, Nagarajarao H, Taylor JK, et al. (2010) The relation of $\mathrm{C}$-reactive protein to chronic kidney disease in African Americans: the Jackson Heart Study. BMC Nephrol 11: 1.

35. Ferreira TS, Lanzetti M, Barroso MV, Rueff-Barroso CR, Benjamim CF, et al. (2014) Oxidative Stress and Inflammation Are Differentially Affected by Atorvastatin, Pravastatin, Rosuvastatin, and Simvastatin on Lungs from Mice Exposed to Cigarette Smoke. Inflammation.

36. Takagi H, Umemoto T (2014) A meta-analysis of randomized head-to-head trials for effects of rosuvastatin versus atorvastatin on apolipoprotein profiles. Am J Cardiol 113: 292-301.

37. Walldius G, Jungner I (2004) Apolipoprotein B and apolipoprotein A-I: risk indicators of coronary heart disease and targets for lipid-modifying therapy. J Intern Med 255: 188-205.

38. Goldberg A, Hammerman H, Petcherski S, Zdorovyak A, Yalonetsky S, et al. (2005) Inhospital and 1-year mortality of patients who develop worsening renal function following acute ST-elevation myocardial infarction. Am Heart J 150: 330-337.

39. Ando G, Morabito G, de Gregorio G, Trio O, Saporito F, et al. (2013) Age, glomerular filtration rate, ejection fraction, and the AGEF score predict contrast-induced nephropathy in patients with acute myocardial infarction undergoing primary percutaneous coronary intervention. Catheter Cardiovasc Interv 82: 878-885. 